\title{
Author's response to letter to the editor: "Fungal otitis externa and wet ear with mucopurulent should be a influencing factors on tympanic membrane closure"
}

\author{
Brett C. A. van Stekelenburg ${ }^{1} \cdot$ Mark C. J. Aarts ${ }^{1}$
}

Received: 3 March 2020 / Accepted: 9 March 2020 / Published online: 24 March 2020

(c) Springer-Verlag GmbH Germany, part of Springer Nature 2020

Dear sir,

We kindly thank you for your response and suggestions concerning our article entitled "Determinants influencing success rates of myringoplasty in daily practice: a retrospective analysis" [1]. We have read your response with great interest.

In your response, the status of the middle ear and external auditory ear canal as influencing factors on the success of myringoplasty are undermined, especially for the chronic tympanic membrane perforations [2, 3]. Despite the retrospective aspect of our study we tried to evaluate as much influencing determinants as possible. We did include the number of ear infections in the year before surgery and the amount of post-operative antibiotics prescribed, which is most likely correlated with the status of the middle ear and external auditory ear canal during or shortly after surgery. However, we did not find any significance concerning these two factors. Therefore, these were ruled out to be of any determining value.

Unfortunately, the nature of the otorrhea was not always assessed, nor was a culture structurally performed in these cases. Therefore we cannot make any statement about the role of mycosis as a determinant for failure of myringoplasty. This remains a point of discussion in current literature and regrettably, we cannot clarify this any further.

Kind regards,

\section{References}

1. van Stekelenburg BCA, Aarts MCJ (2019) Determinants influencing success rates of myringoplasty in daily practice: a retrospective analysis. Eur Arch Otorhinolaryngol 276:3081-3087

2. Nagle SK, Jagade MV, Gandhi SR, Pawar PV (2009) Comparative study of outcome of type I tympanoplasty in dry and wet ear. Indian J Otolaryngol Head Neck Surg 61:138-140

3. Sharma Y, Mishra G, Patel JV (2017) Comparative study of outcome of type i tympanoplasty in chronic otitis media active mucosal disease (wet ear) versus chronic otitis media inactive mucosal disease (dry ear). Indian J Otolaryngol Head Neck Surg 69:500-503

Publisher's Note Springer Nature remains neutral with regard to jurisdictional claims in published maps and institutional affiliations.

This reply refers to the comment available online at https://doi. org/10.1007/s00405-020-05815-0.

Mark C. J. Aarts

mcj.aarts@jbz.nl

1 Department of ENT, Jeroen Bosch Hospital,

's Hertogenbosch, The Netherlands 